\title{
Continuous spinal anesthesia for emergency exploratory laparotomy - A valid approach?
}

\author{
B. MATIAS ${ }^{[1]}$, P. FRAGOSO ${ }^{[2]}$, A. MELO ${ }^{[2]}$ \\ [1] - Department of Anaesthesiology, Centro Hospitalar de Setúbal, EPE - Portugal
}

[2] - Department of Anaesthesiology, Hospital de Braga - Portugal

Centro Hospitalar de Setúbal Hospital de São Bernardo

Hospital Ortopédico Sant'lago do Outão

\section{BACKGROUND}

Emergency exploratory laparotomy in the elderly patient with multiple comorbidities represents a challenge to any anesthetic approach. Continuous spinal anesthesia (CSA) is a centenary technique and, despite it has been unpopular among anesthesiologists ${ }^{1}$, it has several advantages in this context and is now a valid resouce ${ }^{2}$.

\section{CASE REPORT}

81-year old man, with personal history of pulmonary tuberculosis, pleurodesis for chronic pleural effusion and severe chronic obstructive pulmonary disease, proposed for emergency exploratory laparotomy for suspected intestinal obstruction.

CSA was performed in lateral decubitus position, with epidural kit. Catheter was inserted at L2-L3 level, using Levobupivacaine $5 \mathrm{mg}$, Sufentanil $2,5 \mathrm{mcg}$ and Magnesium Sulfate $25 \mathrm{mg}$ (total volume $2 \mathrm{~mL}$ ) to achieve a T4 level block.

The procedure lasted $40 \mathrm{~min}$, during which no additional spinal bolus were necessary, neither sedative medication. Oxygen was given through facial mask and phenylephrine perfusion was needed for hypotension, but stopped at the end of surgery.

Postoperative care at intermediate care unit was uneventful and the patient returned to the ward at day three. Intrathecal catheter was used for postoperative analgesia. The remaining hospital stay was uneventful until discharge.

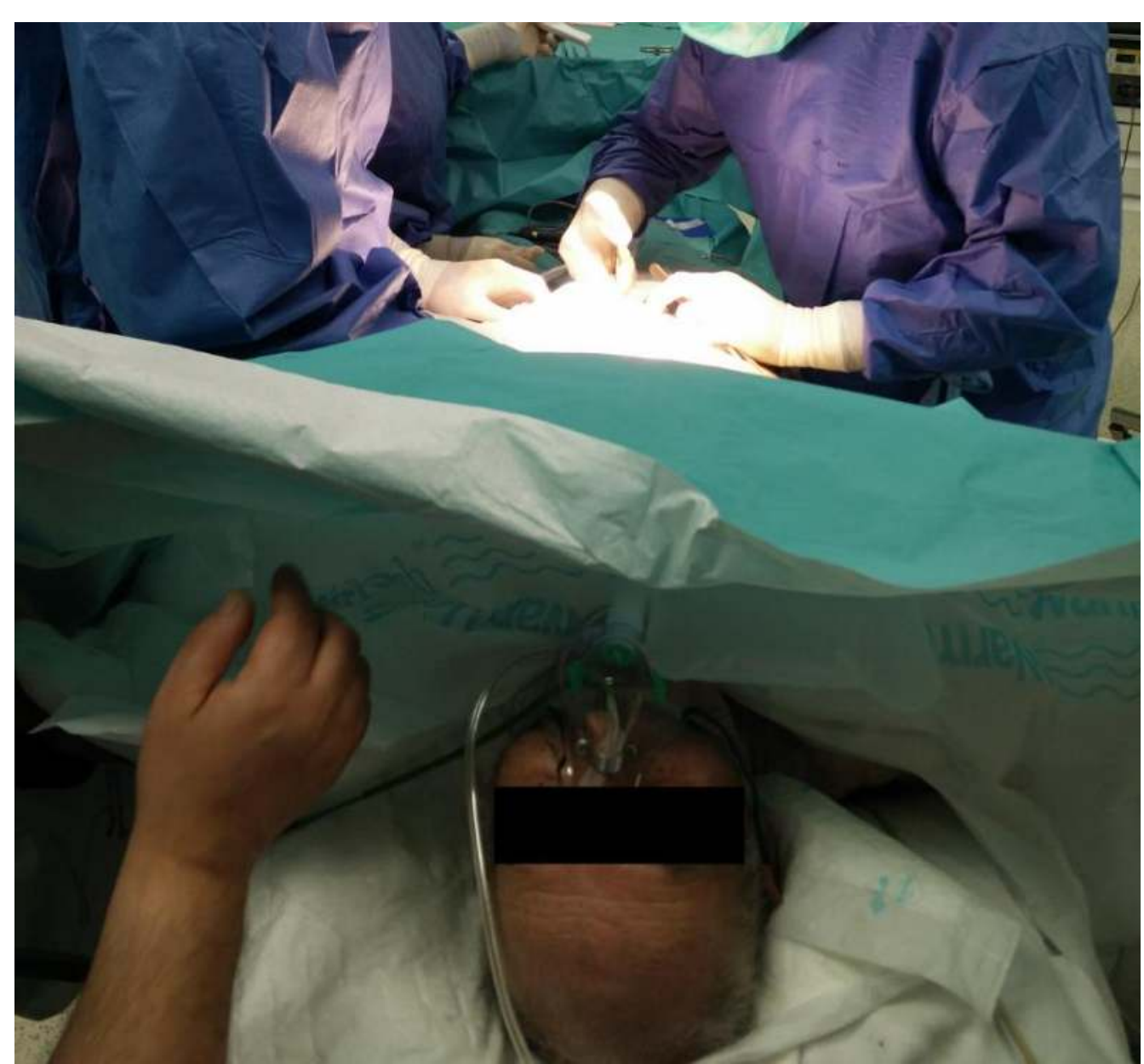

\section{DISCUSSION}

CSA consists on the intermittent administration of small doses of local anesthetic through a catheter placed in the subarachnoid space. Compared to other neuraxial block techniques it has a quicker onset of action, with more pronounced sensory-motor blockade and fewer hemodynamic alterations, as lower doses of local anesthetic are used ${ }^{1,2,3}$. It also allows prolonging anesthesia by the desired time and catheter use for postoperative analgesia ${ }^{1}$. Equipment currently available for CSA ranges from a standard epidural (macrocatheter) to specially designed spinal micro-catheters ${ }^{1}$.

This technique can be chosen as a sole anesthetic technique for laparotomy, especially in patients with severe cardio-respiratory disease who would otherwise be considered unlikely to tolerate general anesthesia or where general anesthesia could result in a prolonged stay in the intensive care unit ${ }^{2}$.

Main concerns regarding CSA are infection, post-dural puncture headache and neurological complications ${ }^{1}$. None of these complications has a frequency that would discourage the use of this type of anesthesia and the latter, historically more relevant, have been considered to be caused by inappropriate technique application ${ }^{2}$.

Our result is in agreement with others already published $^{2,3}$, yet more clinical studies are necessary to reach a consensual approach regarding equipment and dose regimens.

\section{LEARNING POINTS}

Emergency laparotomy in the elderly patient with multiple comorbidities remains a major challenge to any anesthetic approach.

CSA can be used as a sole anesthetic technique, especially in patients with severe cardio-respiratory disease.

\section{REFERENCES}

1-Asian Journal of Pharmaceutical and Clinical Research, 2015; 6:50-53

2-Current Anaesthesia \& Critical Care, 2009; 20:60-64

3-J Anaesthesiol Clin Pharmacol, 2011; 27(1):139-141

Euroanaesthesia

GENEVA 03-05 JUNE 